\title{
Highly efficient modification of beta-lactoglobulin (BLG) gene via zinc-finger nucleases in cattle
}

Cell Research (2011) 21:1638-1640. doi:10.1038/cr.2011.153; published online 13 September 2011

\section{Dear Editor,}

Gene targeting is in widespread use as a gold standard for determining the function of genes in mice and human embryonic stem cells [1]. However, the poor efficiency of this technology has hindered its application to domestic animals, for which embryonic stem cells are not available. Although gene-targeted large domestic animals have been produced successfully by combination of homologous recombination-based targeting strategy and cloning [2-4], the efficiency is very low and, more importantly, the disruption of the targeted gene is usually mono-allelic. It thus takes a long time to obtain a null mutant.

Engineered zinc-finger nucleases (ZFNs) are chimeras of a DNA-specific binding domain $\left(\mathrm{Cys}_{2} \mathrm{His}_{2}\right.$ zinc-finger protein) and a cutting domain (endonuclease Fok I) [5]. The ZFNs induce site-specific DNA double-strand breaks (DSBs) that can be repaired by error-prone non-homologous end-joining (NHEJ) DNA repair or by the errorfree homologous recombination pathway $[6,7]$. The NHEJ repair process often results in targeted mutations in the form of nucleotide deletions or insertions, which can lead to frame-shift mutations. Thus far, ZFN-induced gene editing has been used in the fruit fly Drosophila [6], the nematode Caenorhabditis elegans [8], zebrafish (Danio rerio) [9], cultured mammalian cells [10], rats [11] and pigs [12]. Beta-lactoglobulin (BLG) is a major whey protein in cow's milk and has been shown to be one of the major milk allergens [13]. It is also an ideal locus in mammary gland bioreactors. We report here a successful application of ZFNs on $B L G$ gene knockout in cattle (Figure 1A and 1E). Three pairs of ZFNs were tested in bovine fetus fibroblast cells (Supplementary information, Table S1). The $B L G$-set1 cut the targeting site efficiently and was thus selected for the following work, in which the mRNA of ZFNs was used in order to obtain cell colonies without ZFN plasmid integration. The mutation analysis was done by PCR amplification of the desired site using DNA templates extracted from mixed ZFNstransfected cells and from single colonies (Supplementary information, Figure S1). The PCR products were TA cloned and sequenced to determine the nature of the mutations and targeting efficiency. A total of 96 mutants for the $B L G$ locus from both mixed cells and single colonies were analyzed. Over $80 \%$ of these mutants were short fragment deletions or insertions $(<20 \mathrm{bp})$ and, in some cases, the mutants had both deletions and insertions around the cleavage site (Figure 1B). Earlier work demonstrated that ZFNs spaced 6 bp apart leave mainly 4 bp 5 '-overhangs [14]. In the $B L G$ mutations, 4 bp insertions/ deletions, the typical products of NHEJ repair of ZFNsinduced DSB, are dominant.

High-efficiency disruption was achieved when the ZFNs of $B L G$-set 1 were tested in different bovine cell lines (Supplementary information, Table S2). The mutation efficiency was similar among cell lines, except in cell line 0901, where a single-nucleotide polymorphism in the ZFNs-binding site caused a sharp drop of mutation efficiency. The mRNA of ZFNs worked slightly better than the plasmid but the difference was not significant.

To detect mutations in single-cell colonies, PCR products corresponding to the targeted site were sequenced directly. As shown in Figure 1C, the presence of double peaks after the targeting site in the sequencing curves clearly distinguishes mutants from non-targeted cells. For the $B L G$ gene disruption, 18 mutant singlecell colonies were obtained, and two colonies (16 and 27) contained bi-allelic mutations. Colony 16 (9 and 15 bp deletions) and colony 27 ( 8 and 35 bp deletions) were used as nuclear donors for somatic cell cloning. A total of 8 cloned animals were born (Supplementary information, Table S3), and sequencing results confirmed that they were bi-allelic $B L G$ mutants. However, six of them died soon after birth due to the commonly observed effect of somatic cell cloning (dysfunction of lungs or kidneys) and one died after a month. The remaining animals from colony 16 have lived a healthy life and are now 6 months old (Figure 1E). Nevertheless, the colony 16 (9 and 15 bp deletions, Figure 1D) used in our study likely will not lead to frame shift mutations but should generate two 
A

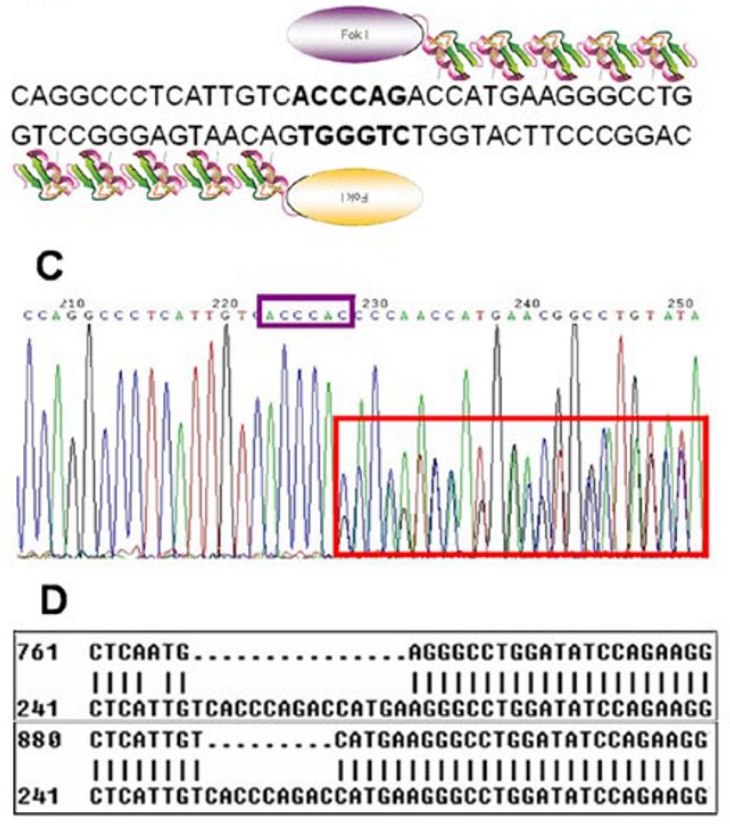

B

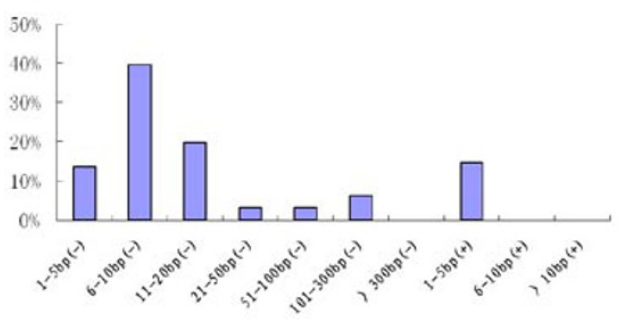

E

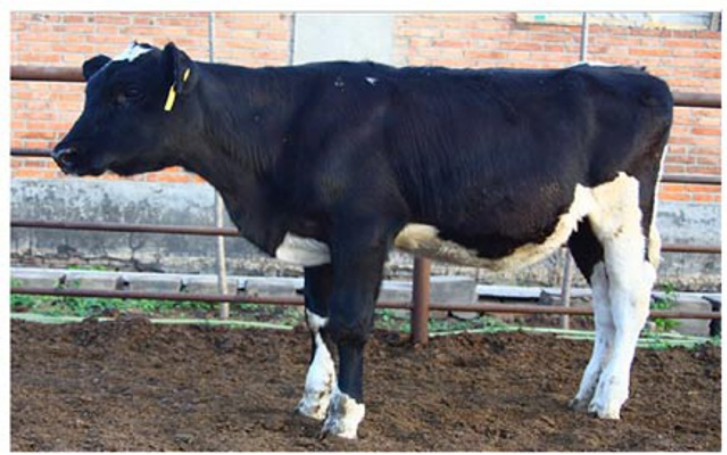

Figure 1 (A) Engineering zinc-finger nucleases for inducing gene disruption at BLG locus in bovine. (B) Nature of ZFNsinduced mutants in bovine fetus fibroblast cells. About 100 mutants were analyzed, and most of them were short fragment deletions or insertions. Vertical axis indicates the mutant percentage. Horizontal axis indicates the different scale of fragment deletion (-) or insertion (+). (C) To detect mutations in a single-cell colony, the PCR products of a single-cell colony were sequenced directly. If the colony is a mutant clone, the peak after (around) ZFNs cutting site (purple box) should be double peaks (red box). (D) Sequence analysis showed that the cloned cattle is a bi-allelic mutant at the BLG locus. (E) The BLG gene-modified cloned cattle derived from colony 16.

shorter versions of the protein compared to the wild-type one. Other colonies containing frame-shift mutations will also be transferred as nuclear donors in the future for further analysis.

To test the fidelity of the ZFNs, we examined the ZFNs-induced mutation efficiency in other species that contain similar sequences at the target sites. Our results showed that the ZFNs are sequence specific (Supplementary information, Table S4). The fact that a one-nucleotide difference in cell line 0901 decreased the cut efficiency of ZFNs dramatically, indicates that the off-target effect of ZFNs was weak in our study.

In summary, this study demonstrates that ZFNs are very efficient for gene editing of large domestic animals as in other species. The high frequency of induced biallelic mutations should save considerable time in the preparation of null mutant animals. Meanwhile, one of the distinguishing features-marker gene-free, would also greatly enhance the commercial potential of using ZFNs in animal production. The results presented here suggest that ZFNs represent a promising approach for specific gene editing in large domestic animals.

\section{Acknowledgments}

We thank Song Li, Haiping Wang, Lili Wang, Rong Li, and Chao Wang (Beijing GenProtein Biotechnology) for excellent technical assistance, Shenglan Cao (Sigma-Aldrich), Yaofeng Zhao, Qingyong Meng and Hongyan Zhao (China Agricultural University) for the manuscript modification. This study was supported in part by the National Major Development Program of Transgenic Breeding (2008ZX08007-001), Major State Basic Research Development Program 973 (2011CBA01000 and 2009CB941000) and the National High Technology Research and Development Program 863 (2010AA10A103 and 2011AA100601).

Shengli $\mathrm{Yu}^{1, *}$, Junjie Luo ${ }^{1, *}$, Zhiyuan Song ${ }^{1}$, Fangrong Ding ${ }^{2}$, Yunping Dai ${ }^{1,2}$, Ning $\mathrm{Li}^{1}$

${ }^{I}$ State Key Laboratory for Agrobiotechnology, College of Biological Science, China Agricultural University, No. 2 Yuanmingyuan West Road, Haidian District, Beijing 100193, China; ${ }^{2}$ Beijing GenProtein Biotechnology Co., Ltd., Room 306, Zhongding Building, Malianwa North Road No. 158, Haidian District, Beijing 100193, China

*These two authors contributed equally to this work. 
Correspondence: Ning Li

Tel: +86-10-62731142; Fax: +86-10-62733904

E-mail: ninglcau@cau.edu.cn

\section{References}

1 Capecchi MR. Gene targeting in mice: functional analysis of the mammalian genome for the twenty-first century. Nat Rev Genet 2005; 6:507-512.

2 Lai L, Kolber-Simonds D, Park KW, et al. Production of alpha-1,3-galactosyltransferase knockout pigs by nuclear transfer cloning. Science 2002; 295:1089-1092.

3 Richt JA, Kasinathan P, Hamir AN, et al. Production of cattle lacking prion protein. Nat Biotechnol 2007; 25:132-138.

4 McCreath KJ, Howcroft J, Campbell KH, et al. Production of gene-targeted sheep by nuclear transfer from cultured somatic cells. Nature 2000; 405:1066-1069.

5 Kim YG, Cha J, Chandrasegaran S. Hybrid restriction enzymes: zinc finger fusions to Fok I cleavage domain. Proc Natl Acad Sci USA 1996; 93:1156-1160.

6 Bibikova M, Golic M, Golic KG, et al. Targeted chromosomal cleavage and mutagenesis in Drosophila using zinc-finger nucleases. Genetics 2002; 161:1169-1175.

7 Lombardo A, Genovese P, Beausejour CM, et al. Gene editing in human stem cells using zinc finger nucleases and integra- se-defective lentiviral vector delivery. Nat Biotechnol 2007; 25:1298-1306.

8 Morton J, Davis MW, Jorgensen EM, et al. Induction and repair of zinc-finger nuclease-targeted double-strand breaks in Caenorhabditis elegans somatic cells. Proc Natl Acad Sci USA 2006; 103:16370-16375.

9 Doyon Y, McCammon JM, Miller JC, et al. Heritable targeted gene disruption in zebrafish using designed zinc-finger nucleases. Nat Biotechnol 2008; 26:702-708.

10 Santiago Y, Chan E, Liu PQ, et al. Targeted gene knockout in mammalian cells by using engineered zinc-finger nucleases. Proc Natl Acad Sci USA 2008; 105:5809-5814.

11 Geurts AM, Cost GJ, Freyvert Y, et al. Knockout rats via embryo microinjection of zinc-finger nucleases. Science 2009; 325:433.

12 Yang D, Yang H, Li W, et al. Generation of PPARgamma mono-allelic knockout pigs via zinc-finger nucleases and nuclear transfer cloning. Cell Res 2011; 21:979-982.

13 Kontopidis G, Holt C, Sawyer L. Invited review: beta-lactoglobulin: binding properties, structure, and function. $J$ Dairy Sci 2004; 87:785-796.

14 Smith J, Bibikova M, Whitby FG, et al. Requirements for double-strand cleavage by chimeric restriction enzymes with zinc finger DNA-recognition domains. Nucleic Acids Res 2000; 28:3361-3369.

(Supplementary information is linked to the online version of the paper on the Cell Research website.) 